\title{
WWP2 promotes degradation of transcription factor OCT4 in human embryonic stem cells
}

\author{
Huiming $\mathrm{Xu}^{1,2}$, Weicheng Wang ${ }^{1,2}$, Chunliang $\mathrm{Li}^{2}$, Hongyao $\mathrm{Yu}^{2}$, Acong Yang ${ }^{1,2}$, Beibei Wang ${ }^{2}$, Ying Jin ${ }^{1,2,3}$ \\ ${ }^{I}$ Shanghai Stem Cell Institute, Shanghai Jiao Tong University School of Medicine, 225 South Chongqing Road, Shanghai 200025, \\ China; ${ }^{2}$ Key Laboratory of Stem Cell Biology, Institute of Health Sciences, Shanghai Institutes for Biological Sciences, Chinese \\ Academy of Sciences/Shanghai Jiao Tong University School of Medicine, Shanghai 200031, China; ${ }^{3}$ Key Laboratory of Cell Differ- \\ entiation and Apoptosis of Chinese Ministry of Education, Shanghai Jiao Tong University School of Medicine, Shanghai 200025, \\ China
}

POU transcription factor OCT4 not only plays an essential role in maintaining the pluripotent and self-renewing state of embryonic stem (ES) cells but also acts as a cell fate determinant through a gene dosage effect. However, the molecular mechanisms that control the intracellular OCT4 protein level remain elusive. Here, we report that human WWP2, an E3 ubiquitin (Ub)-protein ligase, interacts with OCT4 specifically through its WW domain and enhances Ub modification of OCT4 both in vitro and in vivo. We first demonstrated that endogenous OCT4 in human ES cells can be post-translationally modified by Ub. Furthermore, we found that WWP2 promoted degradation of OCT4 through the $26 \mathrm{~S}$ proteasome in a dosage-dependent manner, and the active site cysteine residue of WWP2 was required for both its enzymatic activity and proteolytic effect on OCT4. Remarkably, our data show that the endogenous OCT4 protein level was significantly elevated when WWP2 expression was downregulated by specific RNA interference (RNAi), suggesting that WWP2 is an important regulator for maintaining a proper OCT4 protein level in human ES cells. Moreover, northern blot analysis showed that the WWP2 transcript was widely present in diverse human tissues/organs and highly expressed in undifferentiated human ES cells. However, its expression level was quickly decreased after human ES cells differentiated, indicating that WWP2 expression might be developmentally regulated. Our findings demonstrate that WWP2 is an important regulator of the OCT4 protein level in human ES cells.

Keywords: transcription factor OCT4, WWP2, protein degradation, embryonic stem cells

Cell Research (2009) 19:561-573. doi: 10.1038/cr.2009.31; published online 10 March 2009

\section{Introduction}

Embryonic stem (ES) cells are capable of indefinite self-renewal in vitro and of maintaining the potential to differentiate into derivatives of all three embryonic germ layers. These features give them great potential in both basic research and clinical applications [1]. The pluripotency and self-renewal of ES cells are maintained by several key transcription factors [2]. OCT4 (also called Oct3, encoded by Pou 5f1) is a member of the POU (Pit, Oct,

Correspondence: Ying Jin

Tel/Fax: +86-21-63852591

E-mail: yjin@sibs.ac.cn

Received 11 June 2008; revised 5 September 2008; accepted 25 September 2008; published online 10 March 2009 and Unc) family of transcription factors and is expressed in pluripotent cells, including early embryos, ES cells, embryonal carcinoma (EC) cells, and embryonic germ (EG) cells [3-7]. Expression of OCT4 is downregulated during differentiation. However, it has recently been documented that some malignant cells regain the ability to express OCT4 [8]. The function and regulation of OCT4 expression have been intensively studied in mice. Knocking out the Oct4 gene in mice results in death due to the lack of inner cell mass formation, suggesting that the gene is essential for establishment of pluripotent cell lineages in early mammalian development [9]. Furthermore, it has been shown that a critical amount of Oct4 is required to maintain self-renewal of ES cells. A lower level of expression in ES cells leads to their spontaneous differentiation into trophectoderm cells, whereas elevated 
expression of Oct 4 drives the differentiation into extraembryonic endoderm and mesoderm [10]. In addition, it has been suggested that the abnormalities in the Oct4 expression level might account for the majority of failures observed in somatic cell cloning [11, 12]. Therefore, Oct4 is a master gene in mammalian preimplantation development and is a dose-dependent cell fate determinant. Nevertheless, the mechanisms that regulate Oct4 expression and maintain it within a certain range are not well understood. In particular, little is known about the regulation of Oct4 expression at the post-transcriptional level. Previously, we identified a mouse orthology of human WWP2 (named Wwp2), which specifically interacted with Oct4 and prompted its ubiquitination both in vivo and in vitro [13]. The study indicates that posttranslational modification may play a role in maintaining the Oct4 protein level in ES cells.

The ubiquitin (Ub)-proteasome pathway plays a crucial role in degradation of the proteins involved in many cellular processes [14-17]. Ubiquitination of substrates is mediated through a highly specific enzyme cascade involving the E1 Ub-activating enzyme, E2 Ubconjugating enzyme, and E3 Ub-protein ligase. The E3s are critical components for determining the enzymatic specificity in the Ub cascade, as a result of their direct interaction with substrates. There are two major classes of E3 ligases: the RING finger and the HECT domain E3 ligases $[18,19]$. E6-associated protein (E6-AP), the first identified member of the HECT domain E3 ligases, ubiquitinates p53 [20]. Other members of this family include human neuronally expressed, developmentally downregulated protein 4 (Nedd4) and related Nedd4-like proteins [21]. The family of E6-AP-related E3 ligases is defined by a carboxyl-terminal HECT domain (homologous to the E6-AP carboxyl terminus) of about 350 amino acids. Unlike E6-AP, E3 ligases of Nedd4 family contain 2-4 tryptophan-based WW domains, which consist of 35-40 amino acids and bind certain proteins containing prolinerich motifs. The WW domains of Nedd4 have preference for the consensus sequence PPXY (PY motif) [22, 23]. Human WWP2 (WW domain containing E3 Ub-protein ligase 2) was originally identified by Pirozzi et al. [24] in screening for WW domain-containing proteins. WWP2 contains four tandem WW domains, a C-terminal HECT domain associated with Ub-protein ligase activity, and an N-terminal C2-like domain characteristic of a large family of proteins including protein kinase C. Based on the similarities in structure between Nedd4 and WWP2, it has been suggested that WWP2 belongs to a family of Nedd4-like proteins. With yeast two-hybrid and in vitro binding assays, Wood et al. [25] demonstrated that WWP2 bound to atrophin-1, and they designated WWP2 as atrophin-1 interacting protein 2. Then, McDonald et al. [26] found that WWP2 bound to and inhibited the epithelial $\mathrm{Na}^{+}$channel. Furthermore, it was shown that a catalytically inactive form of WWP2 increased the level of a voltage-gated $\mathrm{ClC}-5$ chloride channel at the cell surface [27]. Nevertheless, it remains to be determined whether WWP2 has an Ub-protein ligase activity and which proteins are its substrates. Our previous study provides strong evidence that Wwp2 is an authentic E3 ligase of Oct4 and is the first protein known to post-translationally modify Oct4 protein. However, it is unclear whether such modification indeed occurs on endogenous Oct4 and whether the Wwp2 expression level affects the Oct4 protein level in ES cells. On the other hand, growing evidence suggests that there are differences between human and mouse ES cells, although they are similar in many ways $[7,28]$. For example, leukemia inhibitory factor, which is essential for maintaining mouse ES cell selfrenewal in culture, does not facilitate the maintenance of undifferentiated human ES cells $[29,30]$. Therefore, further studies on the similarities and differences in Oct4 and related proteins between human and mouse ES cells are necessary for understanding the mechanisms by which Oct4 controls ES cell fates.

To understand how the OCT4 protein level is controlled and whether WWP2 targets OCT4 for ubiquitination and degradation in human ES cells, we studied and characterized OCT4 ubiquitination and degradation in human ES and EC cells. In this article, we report that, like Wwp2, WWP2 specifically interacted with OCT4 and promoted its ubiquitination both in vivo and in vitro. In addition, we demonstrated that WWP2 enhanced OCT4 degradation through the $26 \mathrm{~S}$ proteasome in a dosage-dependent manner. Our data show that the endogenous OCT4 in human ES cells can be post-translationally modified by Ub and that downregulation of WWP2 expression significantly enhances the OCT4 protein level in human ES cells. The study reveals that WWP2 may serve as an important regulator of the OCT4 protein level in human ES cells.

\section{Results}

WWP2 specifically interacts with OCT4 both in vitro and in vivo

We cloned the OCT4 cDNA sequence from human ES cells, which shares $82 \%$ sequence identity with mouse Oct4 [4]. The $W W P 2$ cDNA that we cloned from the HEK 293 cells is $64 \%$ identical to the mouse $W w p 2$ cDNA sequence $[13,24]$. To clarify the interaction between WWP2 and OCT4, GST pull-down assays were performed. As shown in Figure 1A, immobilized GST- 

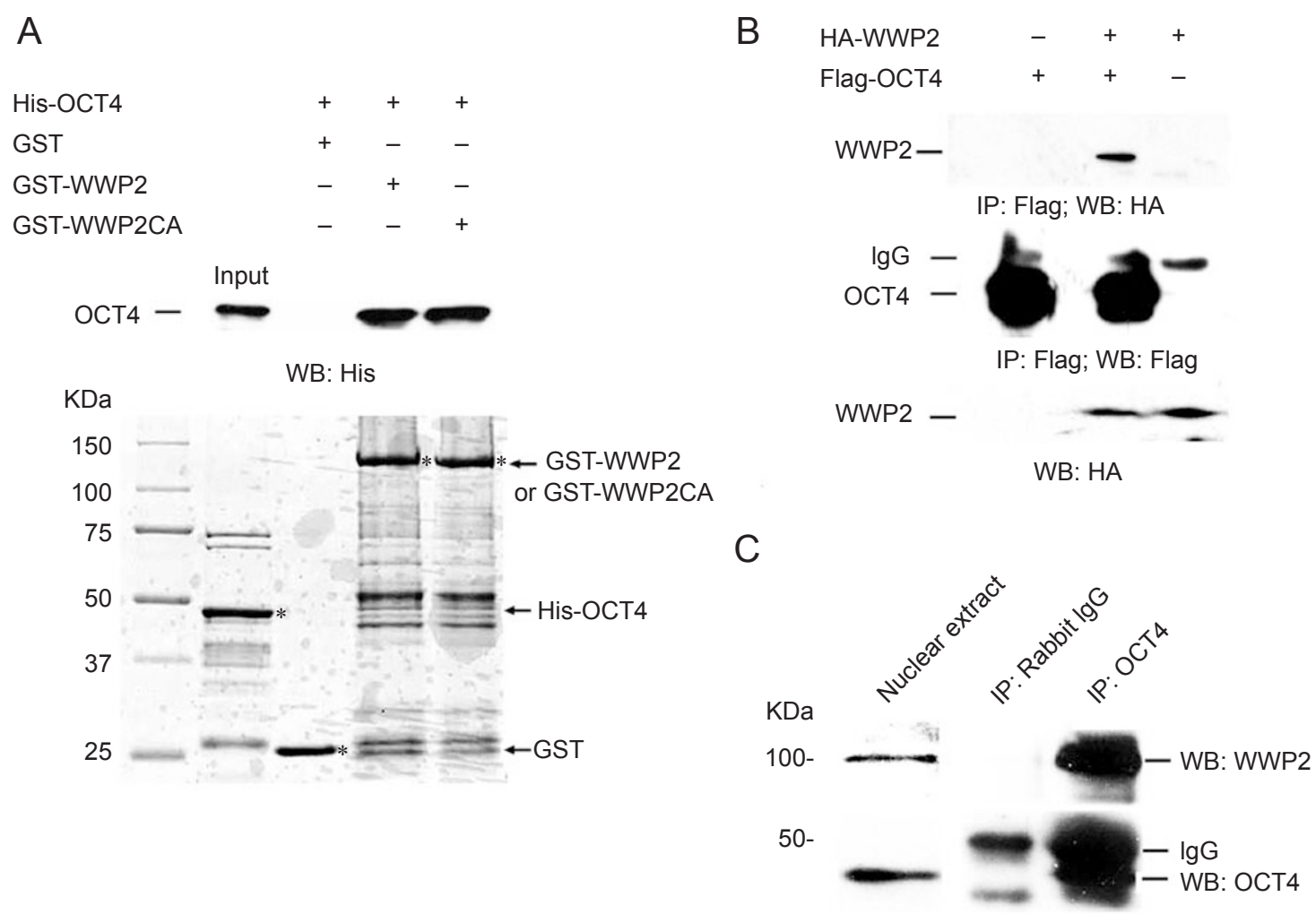

Figure 1 WWP2 associates with OCT4 specifically both in vitro and in vivo. (A) GST pull-down experiments for WWP2 and OCT4. Bacterially expressed and purified GST or GST fusion proteins immobilized on glutathione-sepharose 4B beads were incubated with $200 \mathrm{ng}$ purified His-OCT4 protein. Associated His-OCT4 proteins were blotted with anti-His antibody (top panel). The purified proteins were separated on an SDS-PAGE gel and stained by Coomassie blue (bottom panel). WB, western blotting. * denotes the major band of expressed proteins. (B) Co-immunoprecipitation of OCT4 and WWP2 in HEK 293 cells. Plasmids encoding Flag-OCT4 and HA-WWP2 were cotransfected into HEK 293 cells. Whole-cell lysates were immunoprecipitated with anti-Flag antibody and analyzed by western blotting with an anti-HA antibody (top panel). Cell lysates were also subjected to immunoblotting with anti-Flag and anti-HA antibodies (middle and bottom panels). (C) Association of endogenous OCT4 with WWP2 in human ES SHhES1 cells. The nuclear extract of SHhES1 cells was subjected to immunoprecipitation (IP) with rabbit polyclonal anti-human OCT4 antibody or control rabbit IgG. The immunoprecipitates were analyzed by western blotting with an anti-WWP2 antibody.

WWP2, but not GST alone, was able to pull down HisOCT4, providing evidence for the direct interaction between WWP2 and OCT4 in vitro. In addition, a mutated form of WWP2 (WWP2CA), with a cysteine residue at 838 position changed to alanine, was included in the GST pull-down assays because the cysteine residue in the HECT domains of E3 ligases of this family is known to be highly conserved and essential for their enzymatic activity $[31,32]$. Our data show that the mutation of the cysteine residue at position 838 did not affect the interaction between WWP2 and OCT4 (Figure 1A, lane 4 in the top panel). To further determine whether WWP2 associated with OCT4 in vivo, ectopically expressed HAWWP2 and Flag-OCT4 were co-immunoprecipitated in HEK 293 cells with anti-Flag antibody. As shown in Figure 1B, HA-WWP2 was co-immunoprecipitated by Flag-
OCT4. In order to examine whether endogenous WWP2 and OCT4 exist in the same protein complex, co-immunoprecipitation experiments were performed with nuclear extracts from human ES cells. Endogenous WWP2 was detected in OCT4 antibody-precipitated protein complexes (Figure 1C), but not the control antibody. The result clearly demonstrates the existence of endogenous OCT4-WWP2 complexes in human ES cells. Therefore, we conclude that WWP2 and OCT4 specifically associate with each other both in vitro and in vivo.

The second $w w$ domain of WWP2 is sufficient for its binding to OCT4

As shown in Figure 2A, WWP2 contains three domains: a $\mathrm{C} 2$ domain, a WW domain (including four tandem ww domains), and a HECT domain. To investigate 
which domain of WWP2 interacts with OCT4 using GST pull-down assays, the three domains and the full length of WWP2 were expressed as GST fusion proteins, which are shown in Figure 2B (bottom panel). As shown in Figure 2B (top panel), the WW domain was found to associate with OCT4 by immunoblotting with an anti-His antibody. However, the $\mathrm{C} 2$ and the HECT domains were not capable of binding to OCT4. Therefore, the interaction with OCT4 is probably mediated through the WW domain of WWP2. To further observe which of the four tandem ww domains of WWP2 binds OCT4, GST fusion proteins containing individual ww domains of WWP2 were prepared; GST pull-down experiments with HisOCT4 indicate that the second ww domain (ww2) was the main domain mediating the association between the two proteins, although there was minimal signal between the three other ww domains and OCT4 (Figure 2C). The expression of the GST fusion proteins is shown in Figure 2C (bottom panel). Therefore, the second ww domain of WWP2 was sufficient to mediate the association between WWP2 and OCT4.

\section{Ubiquitination of OCT4}

To investigate the possibilities that OCT4 can be ubiquitinated and WWP2 is an authentic Ub-protein ligase, we conducted an in vitro ubiquitination assay. As shown in Figure 3A (left panel), high molecular weight species, indicating addition of Ub molecules to OCT4, were detected with an anti-Ub antibody only when E1, E2 (UbcH6), Ub, and WWP2 were included in the reaction mixture (lane 4). Of note, the ubiquitination of OCT4 was entirely dependent on the presence of WWP2, and a cysteine residue in the HECT domain of WWP2 is necessary, because the ubiquitination signal in OCT4 vanished when either the cysteine residue at 838 position of WWP2 was mutated to alanine (lane 5) or WWP2 was absent (lane 7). The ubiquitination signal detected when OCT4 was not present (lane 6) could be due to Ub modification of other components in the reaction mixtures. To further verify that signal of the Ub modification was from OCT4, western blotting was conducted with an anti-OCT4 antibody (Figure 3A, right panel). Similarly, ubiquitinated OCT4 can be found only in the presence of E1, E2, Ub, His-OCT4, and wild-type WWP2 (lane 4). No signal was detected when OCT4 was not included in the reaction, confirming that the ubiquitinated protein was from OCT4. Fewer Ub molecules attached to OCT4 were detected by an anti-OCT4 antibody (lane 4 in Figure $3 \mathrm{~A}$, right panel) than by an anti-Ub antibody (lane 4 in Figure 3A, left panel); this may be a result of the steric hindrance by Ub preventing the OCT4 antibody from recognizing all forms of ubiquitinated OCT4. Our results provide strong evidence that WWP2 is an authentic Ubprotein ligase of OCT4.

We next examined whether WWP2 can promote OCT4 Ub modification in vivo. The in vivo ubiquitination

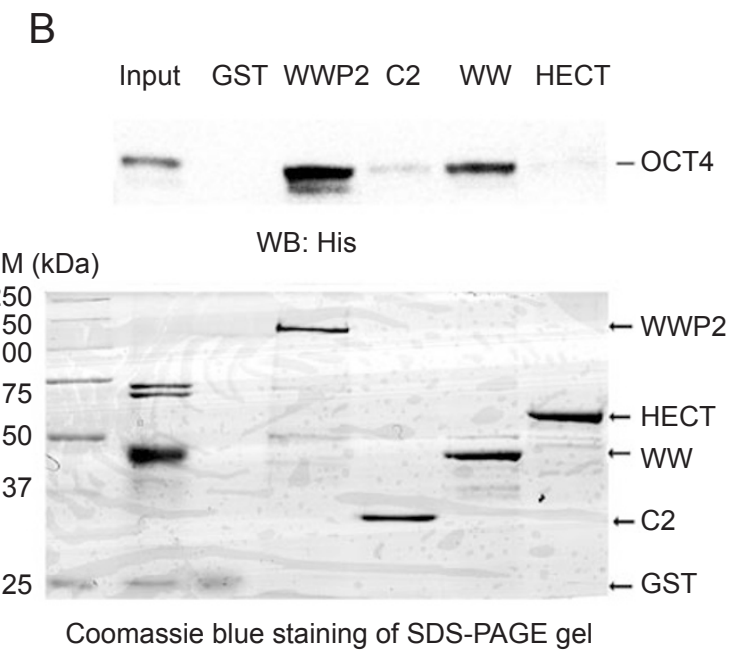

Figure 2 Mapping domains of WWP2 which bind to OCT4. (A) Schematic representation of the modular architecture of the WWP2 coding sequence. (B) GST pull-down experiments for investigating the interaction between the truncated domains of WWP2 and OCT4. The purified GST fusion proteins bound to His-OCT4 were detected with anti-His antibody (top). Bacterially expressed and purified proteins were separated on an SDS-PAGE gel and stained with Coomassie blue (bottom panel). (C) The ability of individual ww domains of WWP2 bound to His-OCT4 was tested by GST pull-down assays and immunoblotting with anti-OCT4 antibody (top panel). GST or GST-fusion proteins of individual ww domains of WWP2 were separated and stained with Coomassie blue (bottom panel). 
A

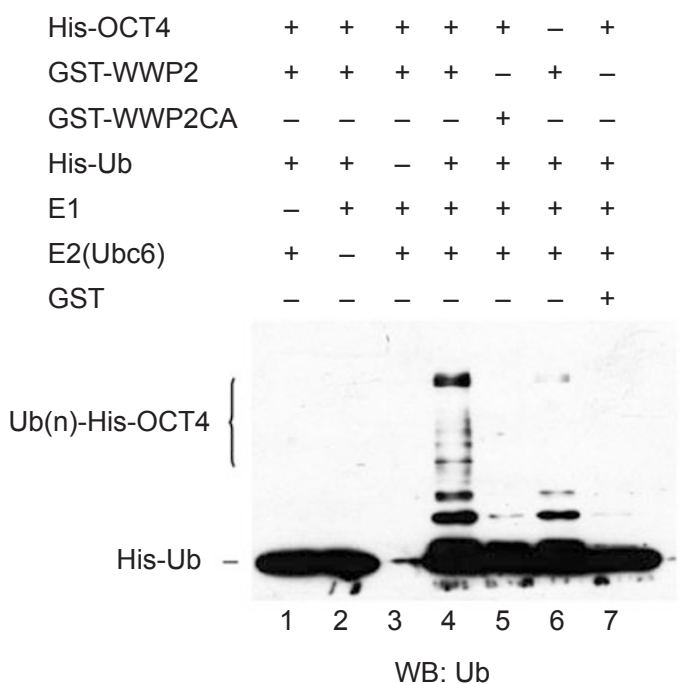

B

$\begin{array}{llll}\text { His-Ub } & + & + \\ \text { OCT4 } & + & + \\ \text { WWP2 } & - & + \\ \text { WWP2CA } & - & - & +\end{array}$

$+\quad+\quad+\quad+$

$++++-+-$

$-\quad-\quad+--$

$++-+++$

$-+++++$

$+-++++$

$-\quad-\quad-\quad+\quad \mathrm{M}(\mathrm{KDa})$

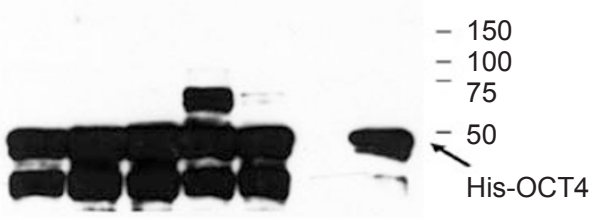

$\begin{array}{ccccccc}12 & 3 & 4 & 5 & 6 & 7 \\ \text { WB: OCT4 } & & \end{array}$
C

$\mathrm{kDa}$

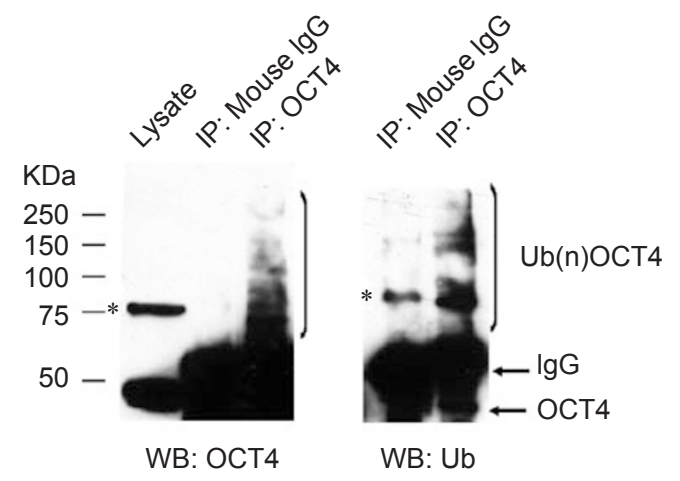

Figure 3 OCT4 is subject to ubiquitination both in vitro and in vivo. (A) OCT4 ubiquitination in vitro assay. Various purified proteins, as indicated, were incubated in the ubiquitination buffer. Ubiquitinated OCT4 was visualized by western blotting with an anti-Ub antibody (left panel) and OCT4 (C10, right panel). (B) HEK 293 cells were transfected with expression vectors, as indicated. The cells were treated with MG132 at $30 \mu \mathrm{M}$ for $5 \mathrm{~h}$ before harvest. Ubiquitinated proteins were isolated by nitrilotriacetic acid affinity beads and analyzed by western blotting with an antibody against OCT4 (top panel). Cell lysates were measured by western blotting with antibodies against WWP2 (middle panel) and OCT4 (bottom panel). Ni, nickel. (C) Ubiquitination of endogenous OCT4 in human ES cells. Whole-cell lysates from human ES cells (HUES-7) were immunoprecipitated with an antibody against OCT4 (C10) or control IgG. The precipitated proteins were analyzed by western blotting with an antibody against OCT4 or Ub. * denotes the nonspecific signal.

experiments were performed with HEK 293 cells transfected with plasmids encoding His-tagged Ub, OCT4, and WWP2. The cells were treated with the proteasome inhibitor (MG132) before harvest. Coexpression of OCT4 with His-Ub resulted in ubiquitination of OCT4 in the high molecular weight forms (Figure 3B, top panel, lane 1), which could be catalyzed by endogenous WWP2 or other E3 enzymes present in HEK 293 cells. Notably, the extent of OCT4 ubiquitination was clearly increased (lane 2) when WWP2 was coexpressed. In contrast, the level of OCT4 ubiquitination in the presence of WWP2CA was even lower than that in the absence of wild- 
type WWP2 (comparing lanes 3 and lane 1), suggesting that WWP2CA acted in a dominant-negative manner. The protein levels of WWP2 and OCT4 in whole-cell lysates are shown in the lower panels of Figure 3B. This result indicates that WWP2 can promote OCT4 ubiquitination in mammalian cells and that the cysteine residue in the HECT domain of WWP2 plays an essential role in the reaction. Lastly, we tested whether the endogenous OCT4 in human ES cells is modified by Ub under physiological conditions. As shown in Figure 3C, higher mo- lecular weight forms of ubiquitinated OCT4 were evident in the OCT4 antibody-precipitated complexes and were detected by both OCT4 and Ub antibodies (Figure 3C). In contrast, no ubiquitinated OCT4 was detected in the control antibody precipitates. This result demonstrates that endogenous OCT4 in human ES cells is subject to post-translational modification by $\mathrm{Ub}$. Taken together, these data indicate that human OCT4 can serve as a substrate for WWP2-mediated ubiquitination both in vitro and in vivo, and that the active site cysteine residue in
A

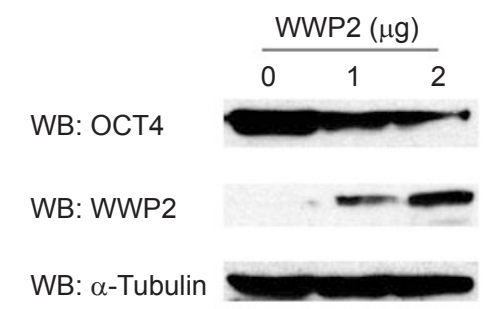

B
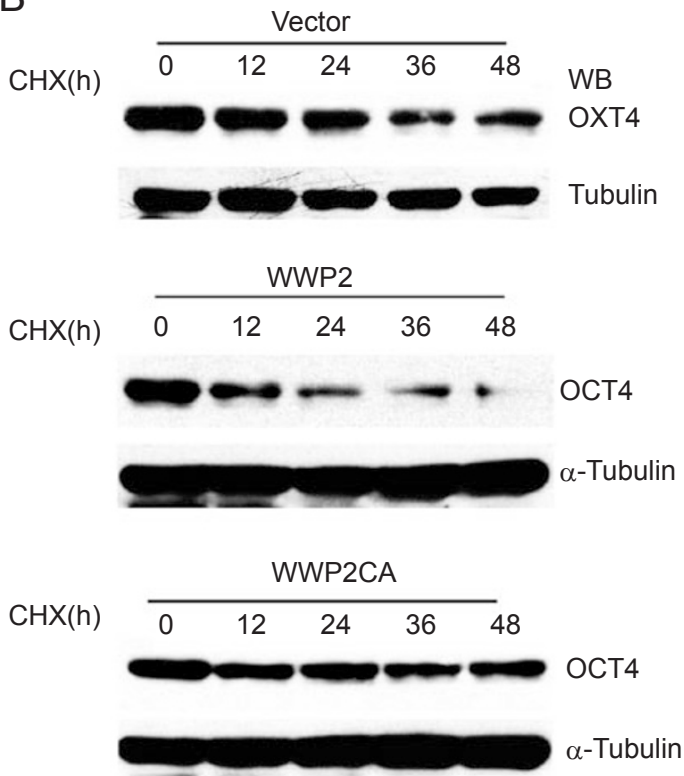

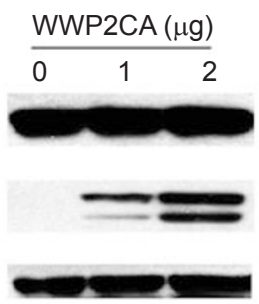

C
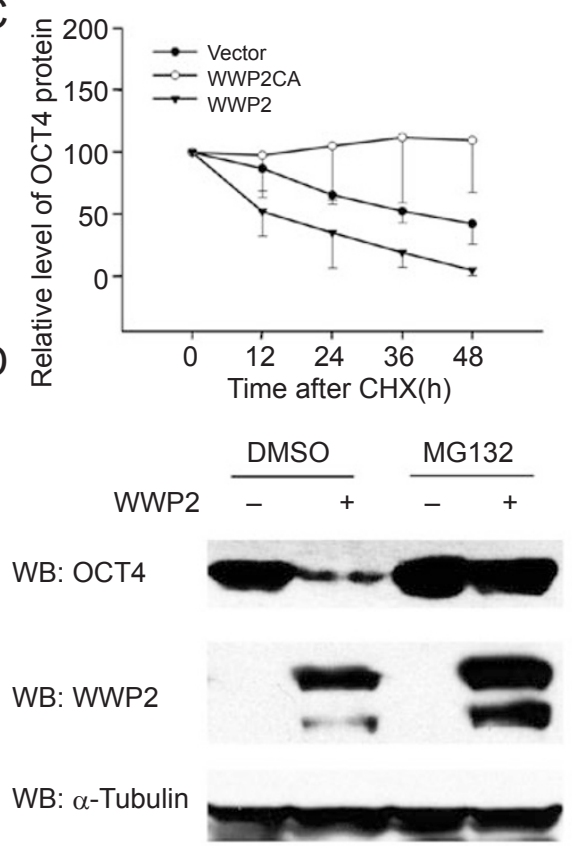

Figures 4 WWP2 promotes degradation of OCT4 through 26S proteasomes. (A) WWP2 reduces the steady-state level of OCT4 protein in a dosage-dependent manner. HEK 293 cells were transfected with constant amount of OCT4 (0.5 $\mu \mathrm{g})$ together with increasing amounts of WWP2 or WWP2CA expression vectors. After $48 \mathrm{~h}$, cells were lysed, and cell lysates were measured by immunoblot with antibodies against OCT4 (top panel) and WWP2 (middle panel). (B) WWP2 enhances turnover of OCT4 protein. HEK 293 cells were transfected with OCT4 along with WWP2, WWP2CA expression vectors, or the empty vector and treated with cycloheximide $(\mathrm{CHX})$ at a concentration of $15 \mu \mathrm{g} / \mathrm{ml}$ for the indicated time. The OCT4 protein levels at different time points were analyzed and compared by western blotting with anti-OCT4 antibody. (C) The densities of the specific bands from the western blotting in panel (B) were measured by densitometry. The experiments were repeated four times. The error bars represent standard derivation. (D) WWP2 promotes OCT4 degradation through 26S proteasomes. HEK 293 cells were transfected with OCT4 together with WWP2 expression vectors or empty vector. At 36-48 h after transfection, the cells were treated with $30 \mu \mathrm{M}$ of MG132 (Sigma) or DMSO for $5 \mathrm{~h}$ before harvest. Cell lysates were measured by western blotting with antibodies against OCT4 (top panel) or WWP2 (middle panel). In the above experiments, $\alpha$-tubulin was used as the loading control. 
the HECT domain of WWP2 is required for promoting OCT4 ubiquitination, although its mutation does not affect the association of WWP2 with OCT4 (Figure 1A).

WWP2 promotes degradation of OCT4 through the $26 \mathrm{~S}$ proteasome in a dosage-dependent manner

Data from our previous study of mouse Wwp2 and Oct4 suggest that Wwp2 could be involved in regulation of the steady-state level of Oct4 protein [13]. However, the topic has not been well investigated. To further explore the functional relationship between WWP2 and OCT4, we transiently transfected HEK 293 cells with a constant amount of OCT4 along with an increasing amount of WWP2 expression vectors. As shown in Figure 4A (left panel), the OCT4 protein level progressively decreased with the increase in the WWP2 expression level. As a negative control, the steady-state level of $\alpha$ tubulin protein remained unchanged, regardless of the increase in the WWP2 expression level, suggesting the specificity of WWP2 acting on OCT4. Interestingly, the OCT4 protein level stayed at a constant level when the WWP2 expression vector was replaced by WWP2CA (Figure 4A, right panel). The result indicates that WWP2 reduced the OCT4 protein level in HEK 293 cells in a dosage-dependent manner and that its enzymatic activity was required for this function.

To investigate whether the reduction in the protein level of OCT4 in the presence of WWP2 was due to an increase in OCT4 degradation, we analyzed OCT4 turnover by treating the cells with cycloheximide (CHX), an inhibitor of protein synthesis. As shown in Figure 4B, in the vector group (top panel), the OCT4 protein level slowly decreased after the addition of CHX, suggesting that OCT4 is a relatively stable protein in HEK 293 cells. However, in the presence of WWP2, the turnover of OCT4 was substantially enhanced (Figure 4B, middle panel). Little OCT4 protein was detectable at $48 \mathrm{~h}$ after CHX treatment. In contrast, the OCT4 protein level remained relatively stable over the period of $\mathrm{CHX}$ treatment in the presence of WWP2CA (Figure 4B, bottom panel). The result is consistent with the result shown in Figure 4A, implying a dominant-negative function of WWP2CA in regulation of the OCT4 protein level. The quantitative data from four experiments are presented in Figure 4C. These observations clearly show that WWP2 promotes degradation of the OCT4 protein in human cells. To further characterize the role of WWP2 in control of OCT4 protein degradation, we examined whether WWP2-mediated OCT4 degradation occurred via the 26S proteasome. We found that the effect of WWP2 in reducing the OCT4 protein level was entirely abrogated when the cells were treated with MG132 before har- vest, although WWP2 reduced the OCT4 protein level drastically in the absence of MG132 (Figure 4D). This result confirms that WWP2 enhances OCT4 degradation through the $26 \mathrm{~S}$ proteasome.

\section{The WWP2 expression level is inversely related to the OCT4 protein level}

It is desirable to learn whether the endogenous OCT4 protein level is affected by the expression level of WWP2 in human ES and EC cells. Human EC cells are derived from teratocarcinomas and are considered as malignant counterparts of human ES cells [33]. They closely resemble human ES cells and have often been used in studies of human embryogenesis. Interestingly, we found that the WWP2 protein level in two human EC cell lines, NTERA2 [34] and 2102Ep [35], was much lower than that in two human ES cell lines, H9 and SHhES1 [36]. Inversely, the OCT4 protein level was significantly higher in human EC cells than that in human ES cells (Figure 5A). The phenomenon suggests that WWP2 may negatively control the OCT4 protein level in human EC and ES cells. To confirm this notion, we transiently transfected human EC 2102Ep cells with small RNAi vectors that contained $19 \mathrm{bp}$ of the coding sequence of WWP2 to downregulate WWP2 expression in the cells. To ensure the specificity of the RNAi effect, two independent sequences were selected from the WWP2 coding region and cloned into RNAi vector (pTER) [37]. The efficiency of $W W P 2$ RNAi (2) and $W W P 2$ RNAi (9) cells was evaluated by measuring $W W P 2$ mRNA levels with quantitative real-time PCR (qPCR). As shown in Figure 5B, the mRNA level of WWP2 in the two WWP2 RNAi cells was about $40 \%$ lower than that in control EGFP RNAi cells. Importantly, as shown in Figure 5C, the OCT4 protein level from the cells described above was evidently elevated when $W W P 2$ expression was downregulated by $W W P 2$ RNAi (lane 2 and 3, as compared with lane 1). The signal of OCT4 is barely detectable because of its low protein level in human EC cells. The quantitative data from three experiments are presented in Figure 5D. To further confirm that OCT4 protein level is affected by the expression level of WWP2 in human ES cells, a lentivirus-based vector transduction system was used to downregulate $W W P 2$ in human ES cells. The lentivirusbased vectors contain a specific short hairpin RNA (shRNA) of luciferase (luc) as a control or the mix of three shRNA of $W W P 2$. As shown in Figure 5E, the OCT4 protein level was elevated (lane 2) when the $W W P 2$ level was downregulated by transduction of WWP2 shRNA. The quantitative results from three experiments are presented in Figure 5F. These data demonstrate that WWP2 plays an important role in the control of the OCT4 pro- 
A

WB: WWP2

WB: OCT4

WB: $\alpha$-Tubulin

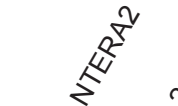

$\overbrace{}^{2}$
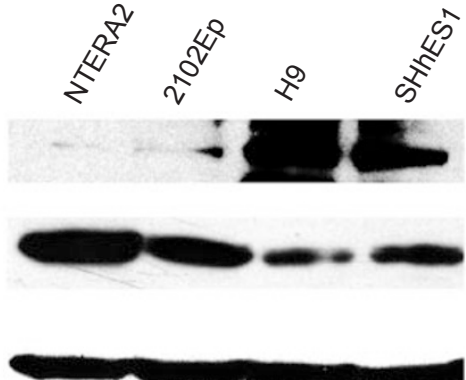

C

RNAi

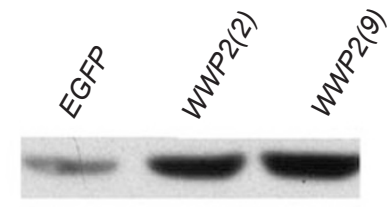

WB: $\alpha$-Tubulin

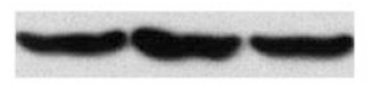

2102Ep cells

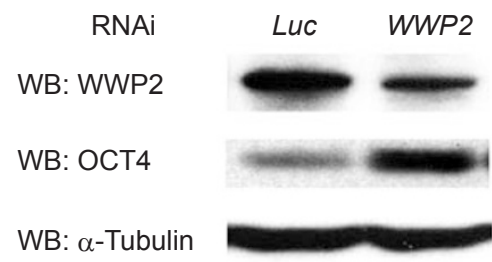

Human ES cells
B
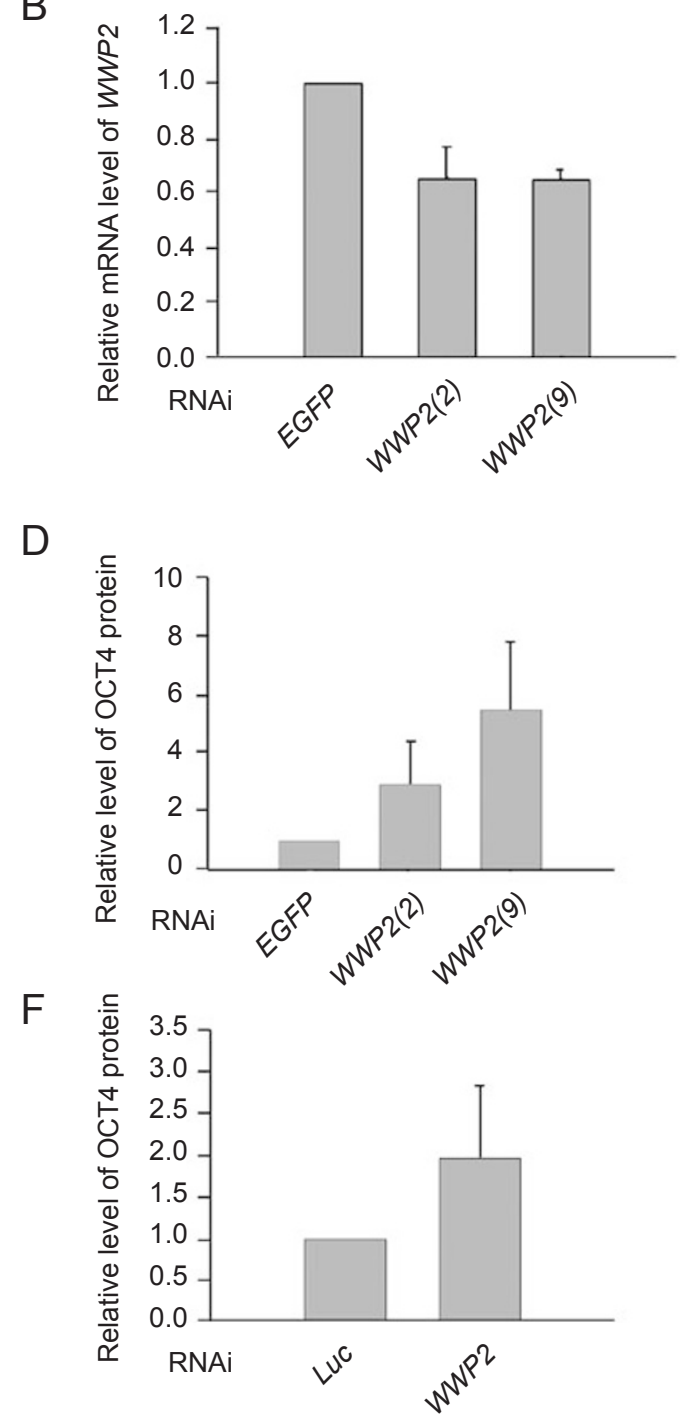

Figure 5 The expression level of WWP2 is inversely related to the OCT4 protein level in human ES and EC cells. (A) Human EC and ES cells differ in their OCT4 and WWP2 protein levels. Cell lysates from the indicated human ES or EC cells were collected and measured by immunoblot with antibodies against WWP2 (top panel) and OCT4 (middle panel). (B) Downregulation of the WWP2 mRNA level by RNAi in human EC cells. Human EC cells (2102Ep) were transfected with EGFP RNAi or WWP2 RNAi (2), or WWP2 RNAi (9) plasmids. The mRNA level of WWP2 was compared using qPCR. (C) Downregulation of the WWP2 mRNA level enhances the steady-state level of OCT4 protein in human EC cells. The protein levels of OCT4 in human EC cells described in panel (B) were measured by western blotting with an antibody against OCT4. (D) The density of the specific bands from the western blotting in (C) was measured by densitometry. The data from the three experiments were quantitatively presented as mean \pm SD. (E) Down-regulation of WWP2 expression by lentivirus-based RNAi vectors enhances the OCT4 protein level in human ES cells (SHhES1). Protein levels of WWP2 and OCT4 were measured by western blotting with antibodies against WWP2 (top) and OCT4 (center), respectively. (F) The density of the specific bands from the western blotting in panel $\mathrm{E}$ was measured by a densitometer, and the data from three experiments were presented as in the panel (D). In the above experiments, $\alpha$-tubulin was used as the loading control.

tein level in both human ES and EC cells.

WWP2 is highly expressed in undifferentiated human ES cells and various human tissues/organs
As a protein important in regulating the OCT4 protein level in human ES cells, what is the expression profile of WWP2 during human ES cell differentiation? We compared the expression of WWP2 in undifferentiated 
A

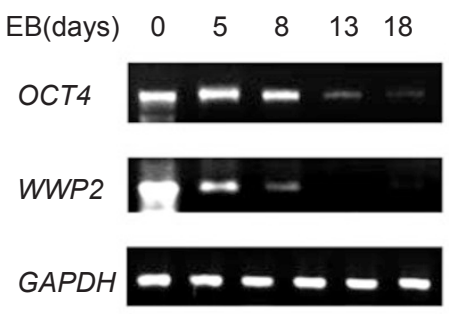

B

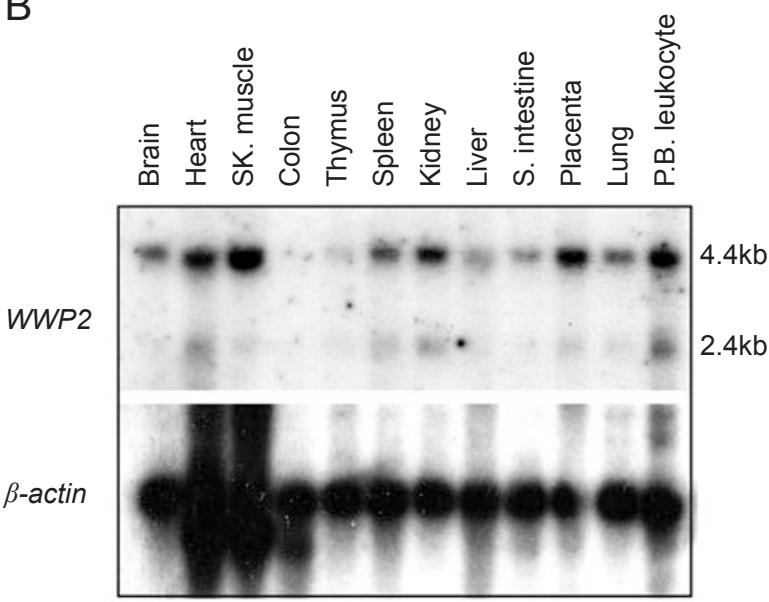

Figure 6 The expression profile of WWP2 in the human ES cell-derived EB model and in various human tissues/organs. (A) Expression of WWP2 is developmentally regulated. Human ES cells (HUES-7) spontaneously differentiated into EBs. Total RNA was extracted at indicated times. Semi-quantitative RTPCR was performed with specific primers for OCT4 and WWP2. The experiments were performed three times, and the representative data are shown. (B) Northern blot analysis of WWP2 transcript in various human tissues/organs with an $\alpha-p^{32}$-labeled cDNA probe of WWP2 (upper panel) and $\beta$-actin (lower panel). SK, skeletal; S, small; PB, peripheral blood.

human ES cells with that in differentiated EBs. RNA was extracted from undifferentiated human ES cells (HUES7) and EBs in suspension at 5, 8, 13 and 18 days, respectively. The expression level of OCT4 and WWP2 was measured by semi-quantitative RT-PCR. As shown in Figure 6A, both OCT4 and WWP2 were highly expressed in undifferentiated human ES cells (day 0). As expected, OCT4 expression decreased gradually with EB formation in a time-dependent manner. Similarly, $W W P 2$ expression was reduced after EB formation. However, it seems that the reduction in $W W P 2$ expression was quicker than that in OCT4. The WWP2 transcript was hardly detectable at day 13 of EB formation, when the OCT4 mRNA was easily detected. Interestingly, a low level of $W W P 2$ signal appeared again at day 18 of EB formation. The observation indicates that WWP2 is a developmentally regulated protein and might be involved with the estab- lishment of pluripotency and cell differentiation during early human development.

The expression pattern of $W W P 2$ during human ES cell differentiation prompted us to further investigate the WWP2 expression pattern in various human tissues/ organs. Although Wood et al. [25] reported a ubiquitous expression of WWP2 in several human organs, the tissues/organs included in their study were limited. To further confirm and expand on their findings, we conducted northern blot analysis with a specific WWP2 probe. As shown in Figure $6 \mathrm{~B}$, a northern blot identified two WWP2 transcripts 4.4 and $2.4 \mathrm{~kb}$ in length. The $4.4-\mathrm{kb}$ transcript was the predominant form. Among the human tissues/organs examined, the WWP2 transcript level was the highest in the skeletal muscle, peripheral leukocytes, placenta, heart, and kidney; higher in the brain, spleen, and lung; and lower in the thymus, liver, colon, and intestine. The result suggests a ubiquitous distribution and involvement of WWP2 in a variety of functions.

\section{Discussion}

The POU domain transcription factor, Oct4, is one of the master regulators that maintain mouse ES in an undifferentiated state and is essential for the establishment of pluripotent inner cell mass in murine embryogenesis. However, little is known regarding human OCT4 compared with murine Oct4. Similar to mouse Oct4 gene, the human $O T F 3$ gene contains five exons. However the human OCT4 gene encodes two isoforms, OCT4A and OCT4B, by alternative splicing. OCT4A and OCT4B have 360 and 265 amino acids, respectively; the 225 amino acids at the C-terminal are identical. In accordance with the data from the study in mouse, human OCT4 expression is much higher in the inner cell mass than in differentiated trophectoderm [6]. Interestingly, unlike mouse Oct4, which is exclusively detected in pluripotent embryonic cells and germ cells, lower levels of expression of both human OCT4A and OCT4B were found in some human tissues $[38,39]$. In addition, it was shown that OCT4 and multiple OCT4 pseudogenes are transcribed in human adult normal tissues and cancers [38, $40,41]$. Therefore, it appears that human OCT4 differs from its murine counterpart, Oct4, with respect to its expression regulation and function. Nevertheless, it is well accepted that OCT4 is essential for maintaining human ES cells in an undifferentiated state [42] and an aberrant expression of OCT4 can lead to development of tumors [43]. A critical unresolved question is how OCT4 expression is regulated in human ES cells. In the present study, the cDNA of human OCT4 was amplified from RNA of undifferentiated human ES cells. The sequence shows 
that the cloned cDNA is $100 \%$ identical to human $O C$ $T 4 A$. We expressed the OCT4 gene in vitro as His-fusion protein and in mammalian cells (Figure 1). Additionally, we demonstrated that OCT4 interacted specifically with a HECT domain Ub-protein E3 ligase, WWP2, both in vitro and in vivo. Furthermore, we demonstrated that (i) endogenous OCT4 in human ES cells can be modified by Ub; (ii) WWP2 enhances OCT4 protein ubiquitination and degradation through the $26 \mathrm{~S}$ proteasome in a dosage-dependent manner; and (iii) downregulation of $W W P 2$ expression evidently elevates the OCT4 protein level in undifferentiated human ES and EC cells. These findings establish that WWP2 is a critical regulator in maintaining a proper OCT4 protein level in human ES cells. Although Wwp2/WWP2 interacts with Oct4/OCT4 in both mouse and human ES cells, it appears that Wwp2 and WWP2 function differently in these two species. Unlike WWP2, the Wwp2 expression level does not affect the Oct4 protein level in undifferentiated mouse ES cells, but it regulates the Oct4 protein level when mouse ES cells are induced to differentiate into certain lineages. Moreover, mouse Wwp2 seems to promote Oct4 degradation only when its expression level is within a narrow range (Liao et al., unpublished data). Therefore, further elucidation of the nature of Wwp2/WWP2 function is required to understand how Oct4/OCT4 expression is tightly controlled.

Human ES cells can reproducibly differentiate in vitro into EBs comprising the three embryonic germ layers [44], which provide a good model to study early human embryonic development, as well as regulation of gene expression. Here, we reported the induction of cystic EBs from human ES cells and characterized OCT4 and $W W P 2$ expression in these EBs. We found that mRNA level of $W W P 2$ was high in undifferentiated human ES cells, but quickly decreased upon ES cell differentiation into EBs, suggesting that $W W P 2$ expression is developmentally regulated and might be involved with the maintenance of ES cell pluripotency. More intriguingly, the reduction in $W W P 2$ expression seems to parallel the decline in OCT4 expression, which is in contrast to our finding that the WWP2 expression level was inversely related to the OCT4 protein level in undifferentiated stem cells (Figure 5). One possible explanation for this discrepancy is that the function of WWP2 in undifferentiated ES cells is different from that in differentiated cell lineages. Of note, $W W P 2$ mRNA appeared again at EB day 18 , implying that it may participate in the differentiation of specific cell lineages and associate with proteins other than OCT4.

In summary, we have isolated OCT4 cDNA from human ES cells and demonstrated that human OCT4 spe- cifically interacted with an E3 Ub-protein ligase, WWP2. WWP2 catalyzed OCT4 Ub modification both in vitro and in vivo and reduced the intracellular OCT4 protein level in a dose-dependent manner. Importantly, we provide evidence that downregulation of $W W P 2$ expression could significantly elevate the OCT4 protein levels in human ES and EC cells. To our knowledge, this is the first demonstration that human OCT4 associates with an Ub-protein ligase and that WWP2 promotes its substrate degradation. The findings identify a new mechanism by which the OCT4 protein level and functions are precisely controlled in human ES cells. Further investigation is required to determine how ubiquitination of OCT4 is regulated and whether WWP2 plays an important role in regulation of proliferation and differentiation of human ES cells.

\section{Materials and Methods}

\section{Constructs}

The OCT4 (XM_175178) and WWP2 (NM_007014) genes were amplified by RT-PCR with RNA of human ES cell line HUES-7 (a kind gift from DA Melton) [28] and human embryonic kidney 293 cells (HEK 293, a kind gift from R Baer), respectively. The cDNAs were cloned into pGEM-T-Easy (Promega) and then subcloned into pGEX-4T-1 (Amersham Biosciences) and pET$30 \mathrm{a}(+)$ (Novagen) vectors for their expression in bacteria. They were subcloned into pCMV-Not (a kind gift from R Baer), pcDNA3 (Invitrogen), and Flag-pPyCAGIP(modified pPyCAGIP) for their expression in mammalian cells. His-Ub vector was a kind gift from D Bohmann. Lentivirus vector LentiLox3.7 was a kind gift from $\mathrm{H}$ Zaehres. The vectors $\mathrm{p} \Delta 8.91$ and VSVG were gifts from $\mathrm{B}$ Reubinoff. Amino acid residual mutants were generated by PCRbased site-directed mutagenesis. Truncated individual ww domains of human WWP2, namely ww1-ww4, were amplified by PCR from full-length $W W P 2$ and then cloned into pGEX-4T-1. The correct sequence of all vectors was verified by DNA sequencing.

\section{Expression of bacterial recombinant fusion proteins}

The recombinant GST and His fusion proteins were expressed in BL21 and BL21 (DE3) host bacteria, respectively. The protein expression was induced by isopropyl- $\beta$-D-thiogalactose (IPTG) and purified using Glutathione Sepharose 4B beads (Amersham Biosciences) or nitrilotriacetic acid His Bind resin (Novagen), according to the methods recommended by the suppliers.

\section{Antibody}

Affinity-purified rabbit polyclonal antibodies against mouse Oct4 and Wwp2 described previously [13] were used in this study to detect human OCT4 and WWP2 on the basis of the high amino acid homology between the two species. Preliminary experiments have shown that the antibodies specifically recognize human OCT4 and WWP2 (data not shown). Monoclonal antibodies against Flag (Sigma), human OCT4 (C10, Santa Cruz Biotechnology), the His (Santa Cruz Biotechnology), HA (Roche), and Ub (P4D1, Cell Signaling) were used for immunoprecipitation and immunoblotting. 


\section{Cell culture and DNA transfection}

HEK 293 cells were cultured under standard conditions and transfected with the calcium phosphate method. Human EC cells (2102Ep, a kind gift from P Andrew, and NTERA2 cl.sp12, a kind gift from S Przyborski) were grown in DMEM supplemented with $10 \%$ FBS and $2 \mathrm{mM}$ glutamine and transfected with Lipofectamine 2000 following the manufacturer's protocol (Invitrogen). Human ES cell lines - H9 (WiCell, USA), HUES-7, and SHhES1 - were maintained on mouse embryonic fibroblasts and propagated as described previously [36]. Human EBs were formed in the differentiation medium (ES cell culture medium with 20\% FBS in the absence of bFGF) and cultured for various periods of time, as indicated.

Co-immunoprecipitation, GST pull-down, and western blotting analysis

For co-immunoprecipitation, cell lysates were prepared in an immunoprecipitation buffer (20 mM Tris- $\mathrm{HCl}, \mathrm{pH} 7.6,150 \mathrm{mM}$ $\mathrm{NaCl}, 0.1 \%$ Nonidet P-40, $10 \mathrm{mM} \mathrm{MgCl}_{2}, 2 \mathrm{mM}$ EDTA, $10 \%$ glycerol, $1 \mathrm{mM} \mathrm{NaF}$, and proteinase inhibitors) and incubated with a specific antibody at $4{ }^{\circ} \mathrm{C}$ overnight, followed by the addition of protein A-sepharose beads (Sigma) for another $2 \mathrm{~h}$. Co-immunoprecipitation experiments of endogenous OCT4 and WWP2 from nuclear extracts of human ES cells, as well as GST pull-down experiments were performed as described previously [13].

\section{Determination of turnover of OCT4}

HEK 293 cells were transfected with $1.5 \mu \mathrm{g}$ of Flag-OCT4, together with $6 \mu \mathrm{g}$ of Flag-WWP2, Flag-WWP2CA, or pPyCAGIP empty vector. At $24 \mathrm{~h}$ after transfection, the cells were passaged and divided into five dishes for collection of the cells at different time points. At $24 \mathrm{~h}$ after replating, the cells were treated with cycloheximide (CHX, $15 \mu \mathrm{g} / \mathrm{ml}$, Sigma), and harvested at the indicated times. The OCT4 protein level was measured with western blotting using an anti-OCT4 antibody.

\section{Ubiquitination assays in vitro and in vivo}

HEK 293 cells were transfected with $2 \mu \mathrm{g}$ of OCT4, $2 \mu \mathrm{g}$ of Flag-WWP2 or Flag-WWP2CA, together with $4 \mu \mathrm{g}$ of His-tagged$\mathrm{Ub}$ and $1 \mu \mathrm{g}$ of pSV- $\beta$-galactosidase expression vectors. At 36-48 $\mathrm{h}$ after transfection, the cells were treated with $30 \mu \mathrm{M}$ of MG132 (Sigma) or DMSO for $5 \mathrm{~h}$ before harvest and then lysed as described previously [13]. The ubiquitinated proteins were affinity purified on nitrilotriacetic acid His Bind resin and analyzed by western blotting with an anti-OCT4 antibody. An in vitro ubiquitination assay was carried out as described previously [13]. The ubiquitinated proteins were visualized by immunoblotting with an antibody against Ub or OCT4.

For determination of endogenous OCT4 ubiquitination, wholecell lysates from human ES cells (HUES-7) were extracted with RIRA buffer containing 1\% SDS and boiled for $10 \mathrm{~min}$. The boiled extract was diluted 10 -fold with RIRA buffer containing $0.1 \%$ SDS and sonicated briefly. After centrifugation, the supernatant was collected and precipitated with an antibody against OCT4 (C10) or mouse control IgG. The precipitated complexes were further analyzed by western blotting using antibody against OCT4 or $\mathrm{Ub}$.

\section{RNA interference}

To construct RNAi vectors for WWP2, two independent 19- bp sequences (5'-CAC CTA CTT TCG CTT TAT A-3' and 5'GGA GTA CGT GCG CAA CTA T-3') within the coding region of WWP2 gene were selected and cloned into the pTER vector (a kind gift from Marc van de Wetering). The RNAi vector for EGFP was made by selecting 19-bp sequences in the coding region of the EGFP gene (5'-GGC TAC GTC CAG GAG CGC A-3'). The human EC cells (2102Ep) were transfected with Lipofectamine 2000 with the plasmid pTER-WWP2 or pTER-EGFP. To make lentivirus RNAi vectors, three oligonucleotides encoding stem-loop structures targeting the WWP2 gene with CAC CTA CTT TCG CTT TAT, CCT GGA GCT GTA CTT CAT, and GAG TAC GTG CGC AAC TAT and one oligonucleotide CTT ACG CTG AGT ACT TCG targeting the luciferase gene were used as controls. These sequences were cloned under the control of the U6 promoter in the vector LentiLox 3.7 [44] and verified by DNA sequencing.

\section{Human ES cell transduction}

Recombinant virus particles were generated by transient cotransfection of lentiviral plasmids (p8.91, VSV-G, and lentiLox3.7 at 2:1:3 mass ratio) into the $293 \mathrm{~T}$ cell line. The supernatant containing the virus particles was collected 48 and $72 \mathrm{~h}$ after transfection and was concentrated by ultracentrifugation to produce virus stocks, which were stored at $-80{ }^{\circ} \mathrm{C}$, as described [30, 44, 45]. The virus particles were used to transduce human ES SHhES1 cells, which were grown in the conditioned medium on Matrigel-coated dishes.

\section{$R N A$ extraction and RT-PCR}

Total RNA from human ES cells (HUES-7) and EBs was extracted in TRIzol Reagent (Invitrogen), and cDNA was reverse transcribed and amplified by RT-PCR. The template used in PCR was normalized relative to the RT-PCR product of GAPDH. The sequences of OCT4 primers were 5'-CAG TAT CGA GAA CCG AGT GAG AGG C-3' (forward) and 5'-GAC CGA GGA GTA CAG TGC AGT GAA G-3' (reverse). The sequences of WWP2 primers were 5'-CGC AAC TAT GAG CAG TGG CA-3' (forward) and 5'-GGT CGT GCG AGT GTT ATG GT-3' (reverse). The sequences of GAPDH primers were 5'-GGA GTC AAC GGA TTT GGT CG-3' (forward) and 5'-TCC TGG AAG ATG GTG ATG GG-3' (reverse).

\section{Northern blot}

A human multiple tissue northern blot was purchased from Clontech and hybridized with an $\alpha$-32P-ATP-labeled cDNA probe of WWP2 (682 bp of 302-983 of NM 025830.3). The equivalent loading of mRNA in each lane was confirmed by hybridization of the same blot with an $\alpha$-32P-ATP-labeled $\beta$-actin cDNA probe.

All experiments were performed at least three times, and the representative data or quantitative data are shown.

\section{Acknowledgments}

We are grateful to Dr DA Melton (Harvard University) for sharing his human ES cells with us. The study was supported by grants from the National High Technology Research and Development Program of China (2006CB943900), the National Natural Science Foundation of China (General Program, 30500088), the Shanghai Jiao Tong University School of Medicine, and the Shanghai Institutes for Biological Sciences, Chinese Academy of Sciences. The study was also supported by the Shanghai Leading Academic 
Deciline Project (S30201).

\section{References}

1 Sinawat S. Potential applications of human embryonic stem cells. J Med Assoc Thai 2007; 90:1253-1258.

2 Niwa H. How is pluripotency determined and maintained? Development 2007; 134:635-646.

3 Scholer HR, Dressler GR, Balling R, et al. Oct-4: a germlinespecific transcription factor mapping to the mouse t-complex. EMBO J 1990; 9:2185-2195.

4 Rosner MH, Vigano MA, Ozato K, et al. A POU-domain transcription factor in early stem cells and germ cells of the mammalian embryo. Nature 1990; 345:686-692.

5 Okamoto K, Okazawa H, Okuda A, et al. A novel octamer binding transcription factor is differentially expressed in mouse embryonic cells. Cell 1990; 60:461-472.

6 Hansis C, Grifo JA, Krey LC. Oct-4 expression in inner cell mass and trophectoderm of human blastocysts. Mol Hum Reprod 2000; 6:999-1004.

7 Ginis I, Luo Y, Miura T, et al. Differences between human and mouse embryonic stem cells. Dev Biol 2004; 269:360-380.

8 Gidekel S, Pizov G, Bergman Y, et al. Oct-3/4 is a dose-dependent oncogenic fate determinant. Cancer Cell 2003; 4:361-370.

9 Nichols J, Zevnik B, Anastassiadis K, et al. Formation of pluripotent stem cells in the mammalian embryo depends on the POU transcription factor Oct4. Cell 1998; 95:379-391.

10 Niwa H, Miyazaki J, Smith AG. Quantitative expression of Oct-3/4 defines differentiation, dedifferentiation or self-renewal of ES cells. Nat Genet 2000; 24:372-376.

11 Boiani M, Eckardt S, Scholer HR, et al. Oct4 distribution and level in mouse clones: consequences for pluripotency. Genes Dev 2002; 16:1209-1219.

12 Donovan PJ. High Oct-ane fuel powers the stem cell. Nat Genet 2001; 29:246-247.

$13 \mathrm{Xu} \mathrm{HM}$, Liao B, Zhang QJ, et al. Wwp2, an E3 ubiquitin ligase that targets transcription factor Oct-4 for ubiquitination. $J$ Biol Chem 2004; 279:23495-23503.

14 Conaway RC, Brower CS, Conaway JW. Emerging roles of ubiquitin in transcription regulation. Science 2002; 296:12541258.

15 Ciechanover A. The ubiquitin-proteasome pathway: on protein death and cell life. EMBO J 1998; 17:7151-7160.

16 Naujokat C, Hoffmann S. Role and function of the 26S proteasome in proliferation and apoptosis. Lab Invest 2002; 82:965980.

17 Mayer RJ. The meteoric rise of regulated intracellular proteolysis. Nat Rev Mol Cell Biol 2000; 1:145-148.

18 Weissman AM. Themes and variations on ubiquitylation. Nat Rev Mol Cell Biol 2001; 2:169-178.

19 Joazeiro CA, Weissman AM. RING finger proteins: mediators of ubiquitin ligase activity. Cell 2000; 102:549-552.

20 Huibregtse JM, Scheffner M, Beaudenon S, et al. A family of proteins structurally and functionally related to the E6AP ubiquitin-protein ligase. Proc Natl Acad Sci USA 1995; 92:5249.

21 Harvey KF, Kumar S. Nedd4-like proteins: an emerging family of ubiquitin-protein ligases implicated in diverse cellular functions. Trends Cell Biol 1999; 9:166-169.
22 Kato Y, Nagata K, Takahashi M, et al. Common mechanism of ligand recognition by group II/III WW domains: redefining their functional classification. J Biol Chem 2004; 279:3183331841 .

23 Murillas R, Simms KS, Hatakeyama S, et al. Identification of developmentally expressed proteins that functionally interact with Nedd4 ubiquitin ligase. J Biol Chem 2002; 277:28972907.

24 Pirozzi G, McConnell SJ, Uveges AJ, et al. Identification of novel human WW domain-containing proteins by cloning of ligand targets. J Biol Chem 1997; 272:14611-14616.

25 Wood JD, Yuan J, Margolis RL, et al. Atrophin-1, the DRPLA gene product, interacts with two families of WW domaincontaining proteins. Mol Cell Neurosci 1998; 11:149-160.

26 McDonald FJ, Western AH, McNeil JD, et al. Ubiquitinprotein ligase WWP2 binds to and downregulates the epithelial $\mathrm{Na}(+)$ channel. Am J Physiol Renal Physiol 2002; 283:F431F436.

27 Flores SY, Debonneville C, Staub O. The role of Nedd4/ Nedd4-like dependant ubiquitylation in epithelial transport processes. Pflugers Arch 2003; 446:334-338.

28 Cowan CA, Klimanskaya I, McMahon J, et al. Derivation of embryonic stem-cell lines from human blastocysts. $N$ Engl $J$ Med 2004; 350:1353-1356.

29 Thomson JA, Itskovitz-Eldor J, Shapiro SS, et al. Embryonic stem cell lines derived from human blastocysts. Science 1998; 282:1145-1147.

30 Gropp M, Itsykson P, Singer O, et al. Stable genetic modification of human embryonic stem cells by lentiviral vectors. Mol Ther 2003; 7:281-287.

31 Huibregtse JM, Scheffner M, Beaudenon S, et al. A family of proteins structurally and functionally related to the E6AP ubiquitin-protein ligase. Proc Natl Acad Sci USA 1995; 92:2563-2567.

32 Huang L, Kinnucan E, Wang G, et al. Structure of an E6APUbcH7 complex: insights into ubiquitination by the E2-E3 enzyme cascade. Science 1999; 286:1321-1326.

33 Przyborski SA, Christie VB, Hayman MW, et al. Human embryonal carcinoma stem cells: models of embryonic development in humans. Stem Cells Dev 2004; 13:400-408.

34 Horrocks GM, Lauder L, Stewart R, et al. Formation of neurospheres from human embryonal carcinoma stem cells. Biochem Biophys Res Commun 2003; 304:411-416.

35 Josephson R, Ording CJ, Liu Y, et al. Qualification of embryonal carcinoma $2102 \mathrm{Ep}$ as a reference for human embryonic stem cell research. Stem Cells 2007; 25:437-446.

36 Sun BW, Yang AC, Feng Y, et al. Temporal and parental-specific expression of imprinted genes in a newly derived Chinese human embryonic stem cell line and embryoid bodies. Hum Mol Genet 2006; 15:65-75.

37 van de Wetering M, Oving I, Muncan V, et al. Specific inhibition of gene expression using a stably integrated, inducible small-interfering-RNA vector. EMBO Rep 2003; 4:609-615.

38 Zangrossi S, Marabese M, Broggini M, et al. Oct-4 expression in adult human differentiated cells challenges its role as a pure stem cell marker. Stem Cells 2007; 25:1675-1680.

39 Roche S, Richard MJ, Favrot MC. Oct-4, Rex-1, and Gata-4 expression in human MSC increase the differentiation efficiency but not hTERT expression. J Cell Biochem 2007; 101:271-280. 
40 Trosko JE. From adult stem cells to cancer stem cells: Oct-4 Gene, cell-cell communication, and hormones during tumor promotion. Ann N Y Acad Sci 2006; 1089:36-58.

41 Suo G, Han J, Wang X, et al. Oct4 pseudogenes are transcribed in cancers. Biochem Biophys Res Commun 2005; 337:10471051.

42 Hay DC, Sutherland L, Clark J, et al. Oct-4 knockdown induces similar patterns of endoderm and trophoblast differentiation markers in human and mouse embryonic stem cells. Stem Cells 2004; 22:225-235.
43 Atlasi Y, Mowla SJ, Ziaee SA, et al. OCT-4, an embryonic stem cell marker, is highly expressed in bladder cancer. Int $J$ Cancer 2007; 120:1598-1602.

44 Zaehres H, Lensch MW, Daheron L, et al. High-efficiency RNA interference in human embryonic stem cells. Stem Cells 2005; 23:299-305.

45 Rubinson DA, Dillon CP, Kwiatkowski AV, et al. A lentivirusbased system to functionally silence genes in primary mammalian cells, stem cells and transgenic mice by RNA interference. Nat Genet 2003; 33:401-406. 